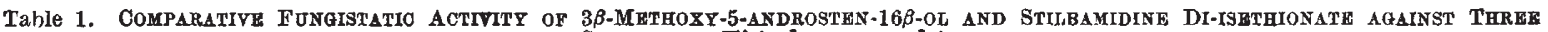
STRAINS OP Histoplatma capsulatum

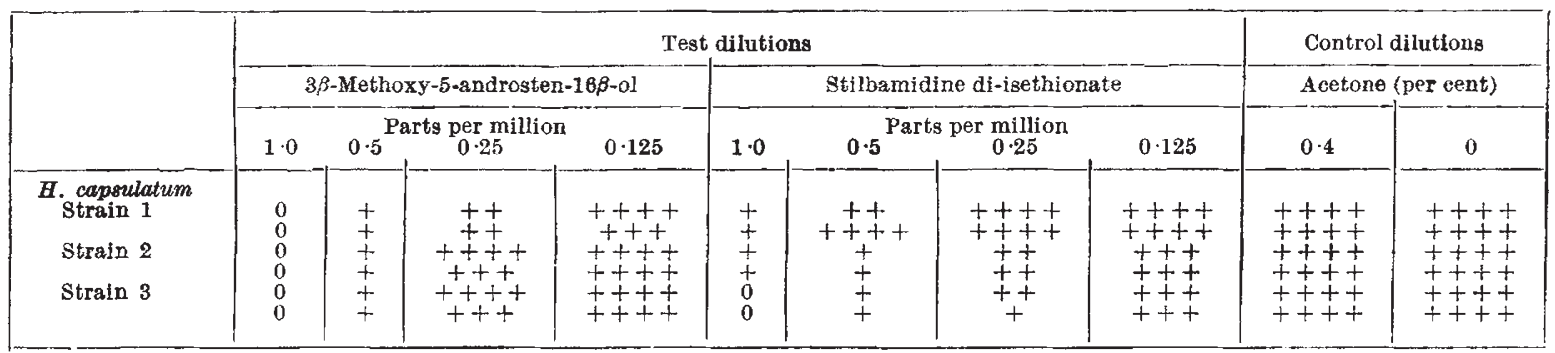

0 , maximally inhibited growth; ++++ , maximal growth

Histoplasma capsulatum tested in 'Difco' Sabouraud's dextrose agar in culture plates ${ }^{5}$, having a fungistatic effect equal to that of stilbamidine di-isethionate.

Table 1 summarizes the comparative effect of

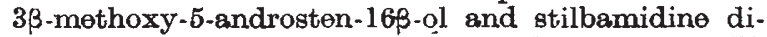
isethionate, assayed simultaneously against $H$. capsulatum.

Curiously, 3-methoxyoestra-1,3,5(10)-trien-16 $\beta$-ol is relatively ineffective against $H$. capsulatum in the culture plate, producing only partial fungistasis at 10 parts per million.

We wish to express our appreciation for the generous support of this research by the Lasdon Foundation, Inc., Yonkers, New York.

$$
\text { R. W. JONES }
$$

Department of Zoology,

Oklahoma State University, Stillwater, Oklahoma.

G. REBELL

Departments of Dermatology and Microbiology, M. N. HuFrman

Department of Biochemistry,

University of Oklahoma School of Medicine, Oklahoma City, Oklahoma.

${ }^{1}$ Jones, R. W., and Huffman, M. N., Trans. Amer. Micro. Soc., 76, 177 (1957).

'Huffman, M. N., Lott, M. H., and Tillotson, A., J. Biol. Chem., $282,447(1956)$

s Huffman, M. N., and Lott, M. H., J. Biol. Chem., 213, 343 (1955).

- Huffman, M. N., Jones. R. W., and Katzberg, A. A., Cancer (in the press).

- Rebell, G., and Lamb, J. H., J. Inveat. Dermat., 21, 331 (1953).

\section{A Colour Reaction of Vitamin E with Vitamin $K$ and Cysteine}

A rew crystals of vitamin $K$ (2-hydroxy-3-methyl1,4-naphthoquinone) and of cysteine (chlorhydrate) are added to a solution containing 7-20 $\mu \mathrm{gm} . / \mathrm{ml}$. of vitamin $\mathbf{E}$ (sodium phosphate of alpha-tocopherol). The solution is heated up to boiling point. About one minute after boiling the solution takes a blue tint. The intensity of colour is proportional to the content of vitamin $E$ and increases during the following three minutes, findlly rendering the solution oprque.

The reaction was not obtained when we tried to replace cysteine by the following substances : cystine, ascorbic acid, sodium hydrosulphite, glutathione, glucuronic acid, methionine, and thiourea.

Nor was the reaction obtained, moreover, when we tried to replace vitamin $K$ by the following compounds : 1,4-naphthoquinone, 2-methyl-1,4-naphtho. quinone, sodium-beta-nsphthoquinone-4-sulphonate ; 2,4-dinitro-1-naphthol-7-sulphonic acid, 2-methyl-1,4- dipropyl-1,4-naphthoquinone, 1,8-dihydroxy-naphthalene-3,6-disulphonic acid, dimethyl-alpha-naphthylamine, alpha-naphthol and bete-naphthol.

The colour reaction is completely inhibited by $p \mathrm{H} 2$ (hydrochloric acid) or $p H \mathrm{H}$ (sodium hydroxide).

If cysteine or vitamin $K$ is added not as crystals but as a solution, even when recently prepared, only an indefinite tint is produced and the solution immediately becomes opaque.

Vitamin $E$ must be the first substance present in the solution and the crystals of cysteine and vitamin $\mathrm{K}$ must be added simultantously before heating. This sequence is fundamental.

Raising the temperature accelerated greatly the completion of the colour reaction, but the latter is produced also without heating.

The solution containing vitamin $\mathrm{E}$, vitamin $\mathrm{K}$ and cysteine exhibits yellow fluorescence, the intensity of which is not apparently proportional to the concentrations of any of the three ingredients. The same fluorescence is produced without vitamin $\mathrm{E}$, but not in the absence of vitamin $K$.

Extraction with organic solvents added to the solution of the three compounds was tried. Ether takes a red tint; the intensity of the colour is apparently proportional to the quantity of vitamin $\mathbf{E}$ present in the solution. The red ether solution gives a blue fluorescence. Petroleum-ether or acetone do not influence the results with ether. A drop of $N / 10$ sodium hydroxide extinguishes the red tint of the supernatant ether almost completely, whereas the blue tint of the original solution takes on a colour between green and yellow, depending on the amount of vitamin E present. Variations of fluorescence are very slight.

Eduardo Cruz-Coke

Institute of Physiological Chemistry, School of Medicine, Universidad de Chile, Av. Borgoño 1470, Santiago, Chile.

\section{Molecular Rearrangement} in the Reaction of Cysteine with I-Fluoro-2,4-Dinitrobenzene

SINCE 1-fluoro-2,4-dinitrobenzene has fungicidal properties ${ }^{1}$ and is useful for the end-group and sequential analysis of proteins, its reaction with cysteine was investigated as part of a study of the mechanism of action of toxicants which function as alkylating agents. In the presence of ten molecular equivalents of cysteine the reaction was first order with respect to the disappearance of 1-fluoro-2,4. dinitrobenzene in dilute aqueous buffer. At $p \mathrm{H} 7 \cdot 0$ 